\section{FRI0651 THE CLINICAL IMPLICATION OF NASAL BIOPSY FOR CLASSIFYING GRANULOMATOSIS WITH POLYANGIITIS IN PATIENTS WITH RHINOSINUSITIS: A SINGLE CENTRE RETROSPECTIVE STUDY}

Juyoung Yoo, Sungsoo Ahn, Seungmin Jung, Jason Jungsik Song, Yongbeom Park, Sangwon Lee. Yonsei university college of medicine, Internal medicine, Seoul, Korea, Rep. of (South Korea)

Background: Granulomatosis with polyangiitis(GPA) mainly involves the upper and lower respiratory tracts and kidneys and induces necrotising vasculitis and granuloma. Nasal biopsy has been recommended in GPAsuspected patients to not only completely cure chronic rhinosinusitis, but also clearly discriminate its aetiologies.

Objectives: We investigated whether the classification of GPA could be made without nasal biopsy in immunosuppressant drug-naïve 45 patients with chronic rhinosinusitis who had previously been classified as GPA.

Methods: We retrospectively reviewed the medical records of 45 patients with GPA. Twenty-six patients exhibited chronic rhinosinusitis, among which 16 patients underwent nasal biopsy (10 with granuloma and 6 without granuloma). We applied the 2007 European Medicines Agency algorithm for the classification of GPA, the 2012 Chapel Hill Consensus Conferences Nomenclature of Vasculitis and the 2017 American College of Rheumatology/European League Against Rheumatism provisional classification criteria for GPA to them for reclassifying GPA. (Figure1)

Results: The mean age was 58.4 years and 17 patients were men. There were no differences in clinical and laboratory results between those with and without granuloma. Among 6 patients without granuloma on nasal biopsy, 3 patients with only ANCAs and chronic rhinosinusitis could be classified as GPA due to PR3-ANCA (or C-ANCA) positivity.

Among 9 patients without nasal biopsy, 3 patients with only chronic rhinosinusitis could be classified as GPA due to GPA-specific lung lesions. (Table 1) When we excluded an item of granuloma in 10 GPA patients with granuloma on nasal biopsy, 4 patients without ANCAs could be classified as GPA due to GPA-specific lung lesions and cartilaginous involvement. (Table 2)


Conclusion: Nasal biopsy is necessary and useful for classifying GPA. However, nasal biopsy could be replaced with PR3-ANCA (or C-ANCA) positivity, GPA-specific lung lesions and cartilaginous involvement in GPA suspected patients with chronic rhinosinusitis.

\section{REFERENCES:}

[1] Jennette JC, Falk RJ, Bacon PA, Basu N, Cid MC, Ferrario F, et al. 2012 revised International Chapel Hill Consensus Conference Nomenclature of Vasculitides. Arthritis Rheum. 2013;65(1):1-11.
[2] Comarmond C, Cacoub P. Granulomatosis with polyangiitis (Wegener): clinical aspects and treatment. Autoimmun Rev. 2014;13(11):1121-5.

Acknowledgement: None

Disclosure of Interests: None declared

DOI: 10.1136/annrheumdis-2019-eular.1895

\section{Epidemiology, risk factors for disease or disease progression}

\section{FRI0652 \\ SERUM CXCL13 LEVELS ARE ASSOCIATED WITH LYMPHOMA RISK AND LYMPHOMA OCCURRENCE IN PRIMARY SJÖGREN'S SYNDROME}

Emmanuella Traianos ${ }^{1}$, James Locke ${ }^{1}$, Dennis Lendrem ${ }^{1}$, Simon J. Bowman ${ }^{2}$, Ben Hargreaves ${ }^{3}$, Victoria Macrae ${ }^{3}$, Jessica Tarn ${ }^{1}$, Wan Fai $\mathrm{Ng}^{1,3}$, UK primary Sjögren's syndrome registry study group. ${ }^{1}$ Newcastle University, Institute of Cellular Medicine, Newcastle upon Tyne, United Kingdom; ${ }^{2}$ University Hospitals Birmingham, Birmingham, United Kingdom; ${ }^{3} \mathrm{NIHR}$ Newcastle Biomedical Research Center, Newcastle upon Tyne, United Kingdom

Background: Primary Sjögren's syndrome (pSS) is an autoimmune disease characterized by an increased risk for non-Hodgkin lymphoma (NHL) development. Ectopic germinal center (GC) formation in the salivary gland (SG) epithelium of pSS patients is thought to be associated with high risk for pSS-associated NHL. The chemokine (C-X-C motif) ligand 13 (CXCL13) is a major chemoattractant for $B$ cell migration into the GC, plays an important role in the organization of $B$ cell follicles, and enhances BCR-triggered $B$ cell activation. The increased B cell activation within ectopic GC may lead to excessive $B$ cell proliferation and along with genetic abnormalities may drive the transition from the early clusters of GC B cells through oligoclonal and later monoclonal B cell expansion to lymphoma.

Objectives: To investigate the potential of $\mathrm{CXCL13}$ as a biomarker to assess $\mathrm{NHL}$ risk in pSS

Methods: Serum CXCL13 concentrations were quantified by ELISA in 48 healthy individuals, 165 pSS patients without NHL and 38 pSS patients with NHL from the United Kingdom Primary Sjögren's Syndrome Registry (UKPSSR). Among these patients, follow-up serum samples from 83 and 11 patients without and with $\mathrm{NHL}$ respectively were available and CXCL13 measured. PSS patients without NHL were stratified into low risk (LR), moderate risk (MR) and high risk (HR) groups according to the lymphoma risk assessment tool proposed by Fragkioudaki et al, 2016. Differences in CXCL13 levels among risk groups were analyzed using the Mann-Whitney test and differences in CXCL13 levels between initial (visit 1) and follow-up (visit 2) samples were measured by repeated measures MANOVA. Associations of CXCL13 with B cell markers were determined by Pearson correlation. Logistic regression analyses were performed to model the NHL risk against CXCL13 concentrations.

Results: At visit 1 , there were $24 \mathrm{LR}, 58 \mathrm{MR}$ and $1 \mathrm{HR}$ pSS patients. Because of the small sample size of the HR "group", it was excluded from further analyses. Serum CXCL13 levels were higher in all pSS groups compared to healthy controls $(p<0.0001)$, and the levels in patients with a history of NHL were higher compared to those without ( $p$ $=0.0311)$. Visit 1 LR patients had significantly lower CXCL13 levels than MR patients $(p=0.0015)$ and pSS patients with NHL $(p=0.0017)$. Serum CXCL13 levels remained stable between visit 1 and visit 2 (mean time between visits $=4$ years) for all pSS groups. CXCL13 was associated with B cell markers, including Immunoglobulin $G(\operatorname{lgG})(p=0.0014)$, B-cell activating factor $(p<0.0001)$, beta-2 microglobulin $(p<0.0001)$, combined free light chains $(p<0.0001), \kappa$ light chain $(p<0.0001), \lambda$ light chain $(p$ $=0.0002)$, $\kappa$-to- $\lambda$ ratio $(p=0.0013)$, and Anti-SSA/Ro autoantibodies $(p=$ 0.0005). Finally, serum CXCL13 levels, age, IgM, IgA, and white blood cell count were independent predictors of $\mathrm{NHL}$ risk score in pSS.

Conclusion: Our findings have demonstrated that serum CXCL13 levels were elevated in pSS patients with NHL and MR pSS patients compared to LR pSS patients and remained stable between visit 1 and visit 2 . CXCL13 was an independent determinant of $\mathrm{NHL}$ risk score and could potentially be used as an additional NHL risk biomarker in pSS. 




*Significant p value $(<0.005)$, " significantp value $(<0.0001)$.

\section{REFERENCES:}

[1] Fragkioudaki S, Mavragani C, Moutsopoulos H. Predicting the risk for lymphoma development in Sjogren syndrome. Medicine. 2016;95(25): e3766.

Table 1. - NHL risk predictors for pSS patients without lymphoma at Visit 1

\begin{tabular}{lcc}
\hline NHL Risk predictors & $\begin{array}{c}\text { Likelihood ratio ChiSquare } \\
\text { Value }\end{array}$ & Prob>ChiSq \\
\hline IgA & 6.6459 & $0.0360^{*}$ \\
IgM & 12.065 & $0.0024^{*}$ \\
Disease duration & 5.4365 & 0.0660 \\
Age & 18.546 & $<.0001^{*}$ \\
Log [White blood cell & 6.1494 & $0.0462^{*}$ \\
count] & & \\
Log $[\mathrm{CXCL13} \mathrm{c(pg/mL)]}$ & 8.3463 & $0.0154^{*}$
\end{tabular}

Disclosure of Interests: Emmanuella Traianos: None declared, James Locke: None declared, Dennis Lendrem: None declared, Simon J. Bowman Grant/research support from: PreviouslyUCB Pharma (to University of Birmingham) and Roche, Consultant for: 2016-7: Novartis, Mitsubishi Tanabe Pharma 2017-8: AstraZeneca, Medlmmune, GFK, Xtlbio, ONO Pharmaceutical 2018-9: Novartis, AstraZeneca, UCB Pharma, Ben Hargreaves: None declared, Victoria Macrae: None declared, Jessica Tarn: None declared, Wan Fai Ng: None declared

DOI: 10.1136/annrheumdis-2019-eular.4134

\section{FRI0653 IMPROVING AND VALIDATING THE PREDICTIVE ACCURACY OF MRI DETECTED SUBCLINICAL INFLAMMATION FOR RHEUMATOID ARTHRITIS DEVELOPMENT IN CLINICALLY SUSPECT ARTHRALGIA}

Xanthe Matthijssen ${ }^{1}$, Fenne Wouters ${ }^{1}$, Debbie Boeters ${ }^{1}$, Aleid Boer ${ }^{1}$,

Yousra Dakkak ${ }^{1}$, Ellis Niemantsverdriet ${ }^{1}$, Saskia Le Cessie ${ }^{1}$, Annette van der Helm - van Mil ${ }^{1,2}$. ${ }^{1}$ Leiden University Medical Center (LUMC), Leiden, Netherlands;

${ }^{2}$ Erasmus University Medical Center, Rheumatology, Rotterdam, Netherlands

Background: Presence of MRI-detected subclinical joint inflammation in clinically suspect arthralgia (CSA) is predictive for progression to Rheumatoid Arthritis. Despite high negative predictive values (94\%); its positive predictive value (PPV) is moderate $(31 \%)$.

Objectives: We studied if, in addition to the presence of inflammation, incorporating information on severity, number and combinations of MRIinflammatory locations improves the predictive accuracy.

Methods: In the discovery cohort, 225 CSA-patients were followed on clinical arthritis development. Contrast-enhanced 1.5T MRIs were made of unilateral $\operatorname{MCP}(2-5)$, wrist and $\operatorname{MTP}(1-5)$-joints at baseline and scored for synovitis, tenosynovitis and bone marrow edema. Severity, number and combination of locations (joint/tendon/bone) with subclinical inflammation were determined, with symptom-free controls of similar age category as reference. Cox regression was used for predictor selection. Predictive values were determined at 1-year follow-up. Results were validated in 266 CSA-patients. Results: In both cohorts $15 \%$ developed arthritis $<1$-year. The number of locations with subclinical inflammation (1-2 locations HR 2.54 (1.11-5.82); $\geq 3$ locations HR 3.75 (1.49-9.48)) and presence of MCP-extensor peritendinitis (HR 4.38 (2.07-9.25)) were independently predictive. Severity and combinations of inflammatory lesions were not. Based on these variables, five risk categories were defined: no subclinical inflammation, 1-2/>3 locations, with/without
MCP-extensor peritendinitis. PPVs ranged 5\% (lowest category; NPV 95\%) $67 \%$ (highest category). Similar findings were obtained in the validation cohort; PPVs ranged 4\% (lowest category; NPV 96\%) - 63\% (highest category). Conclusion: Incorporation of the number of locations with subclinical inflammation and MCP-extensor peritendinitis in MRI-evaluation improved risk stratification, yielding PPVs up to $63-67 \%$. This promotes optimal use of MRI-data in an evidence-based way.

Disclosure of Interests: Xanthe Matthijssen: None declared, Fenne Wouters: None declared, Debbie Boeters: None declared, Aleid Boer: None declared, Yousra Dakkak: None declared, Ellis Niemantsverdriet: None declared, Saskia Le Cessie: None declared, Annette van der Helm - van Mil Grant/research support from: The research leading to these results has received funding from the European Research Council (ERC) under the European Union's Horizon 2020 research and innovation programme (Starting grant, agreement No 714312) and from the Dutch Arthritis Foundation.

The funding source had no role in the design and conduct of the study. DOI: 10.1136/annrheumdis-2019-eular.2612
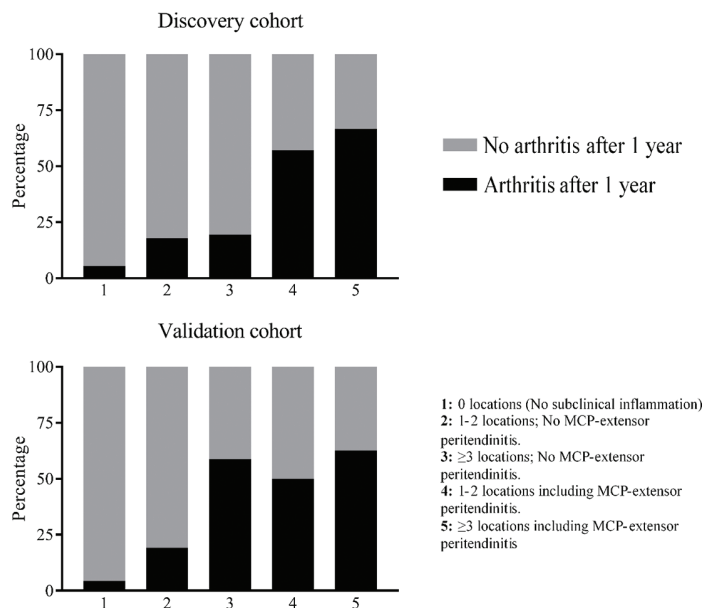

Observed. percentage of patients that developed clinical arthritis in the first year (PPVs in black) per risk category in the discovery and validation cohorts.

\section{FRI0654 DECREASING INCIDENCE OF CARDIOVASCULAR DISEASE IN PATIENTS WITH INCIDENT RHEUMATOID ARTHRITIS IN RECENT YEARS}

Elena Myasoedova ${ }^{1}$, John Davis ${ }^{1}$, Veronique Roger ${ }^{2}$, Sara Achenbach ${ }^{3}$, Cynthia S. Crowson ${ }^{1}{ }^{1}$ Mayo Clinic, Rheumatology, Rochester, MN, United States of America; ${ }^{2}$ Mayo Clinic, Cardiovascular Disease, Rochester, MN, United States of America; ${ }^{3}$ Mayo Clinic, Health Sciences Research, Rochester, MN, United States of America

Background: There is a recognized excess burden of cardiovascular disease (CVD) in patients with rheumatoid arthritis (RA) as compared to the general population. Several studies suggested reduced CVD mortality in RA in recent decades [1-3]. Longitudinal studies on trends in occurrence of CVD events in RA patients over time, and studies comparing trends in CVD events in RA vs general population are lacking.

Objectives: 1) To assess trends in incidence of CVD in patients with incident RA in 1980-2009; and 2) To compare incidence of CVD in RA patients vs non-RA subjects with RA incidence/index date in 2000-09. Methods: The study population comprised Olmsted County, Minnesota residents with incident RA (age $\geq 18$ years, 1987 ACR criteria met in 1980-2009) and non-RA subjects from the same underlying population with similar age, sex and calendar year of index. All subjects were followed until death, migration, or 12/31/2016. Follow-up was truncated for comparability. Incident CVD events included myocardial infarction (MI), stroke (ischemic or hemorrhagic), coronary heart disease (CHD) death and first occurrence of any of these. Patients with CVD events prior to RA incidence/index date were excluded. Cox proportional hazards models were used to compare incident CVD events by decade, adjusting for age and sex. Cumulative incidence of CVD events adjusted for death from other causes was also computed.

Results: The study included 906 patients with RA (mean age 55.9 years; $69 \%$ female). There were 201, 299 and 406 patients with incident RA in 1980-89, 1990-99 and 2000-09, respectively. During median follow-up of 10.6, 10.4 and 10.2 years per decade, CVD events occurred in 31,38 , 